\title{
Criminologie
}

\section{Les effets de la peine sur les proches des contrevenants}

Difficultés et discussion quant à leur problématisation lors de la

détermination de la peine

\section{The effects of punishment on the offenders' relatives}

Difficulties and discussion regarding their problematization at

the sentencing stage

\section{Las consecuencias de la pena sobre los familiares de los detenidos

\author{
Dificultades y discusión en cuanto a su problematización en la \\ etapa de la determinación de la pena
}

\section{Sophie de Saussure,}

Volume 52, numéro 1, printemps 2019

Les proches de personnes judiciarisées : expériences humaines et connaissances carcérales

URI : https://id.erudit.org/iderudit/1059546ar

DOI : https://doi.org/10.7202/1059546ar

Aller au sommaire du numéro

Éditeur(s)

Les Presses de l’Université de Montréal

ISSN

0316-0041 (imprimé)

1492-1367 (numérique)

Découvrir la revue

Citer cet article

de Saussure,, S. (2019). Les effets de la peine sur les proches des contrevenants : difficultés et discussion quant à leur problématisation lors de la détermination de la peine. Criminologie, 52(1), 203-224. https://doi.org/10.7202/1059546ar
Résumé de l'article

Cet article s'intéresse à la problématisation des effets de la peine sur les proches des personnes contrevenantes dans le contexte de la détermination de la peine. Pour observer cet objet, nous aborderons la manière dont le droit criminel moderne organise l'absence des proches des contrevenants, et réfléchirons aux rationalités oeuvrant en coulisse pour construire et sédimenter cette absence. Nous verrons aussi comment s'organisent, en filigrane, des espaces de reconnaissance des effets de la peine sur les proches des contrevenants, et observerons les émergences qui viennent irriter les rationalités disqualifiantes pour ces personnes, rationalités qui dominent encore la pensée et les pratiques en matière pénale. Enfin, nous proposerons une réflexion sur la manière dont le dévoilement des absences et des émergences pourrait contribuer à enrichir et modifier ces rationalités, dans la perspective de construction d'un droit criminel inclusif. 


\title{
Les effets de la peine sur les proches des contrevenants
}

\author{
Difficultés et discussion quant à leur \\ problématisation lors de la détermination \\ de la peine
}

\author{
Sophie de Saussure ${ }^{1,2}$ \\ Candidate au doctorat en droit \\ Chercheuse membre de la Chaire de recherche du Canada \\ en traditions juridiques et rationalité pénale \\ Université d'Ottawa \\ sdesaussure@gmail.com
}

RÉSUMÉ - Cet article s'intéresse à la problématisation des effets de la peine sur les proches des personnes contrevenantes dans le contexte de la détermination de la peine. Pour observer cet objet, nous aborderons la manière dont le droit criminel moderne organise l'absence des proches des contrevenants, et réfléchirons aux rationalités œuvrant en coulisse pour construire et sédimenter cette absence. Nous verrons aussi comment s'organisent, en filigrane, des espaces de reconnaissance des effets de la peine sur les proches des contrevenants, et observerons les émergences qui viennent irriter les rationalités disqualifiantes pour ces personnes, rationalités qui dominent encore la pensée et les pratiques en matière pénale. Enfin, nous proposerons une réflexion sur la manière dont le dévoilement des absences et des émergences pourrait contribuer à enrichir et modifier ces rationalités, dans la perspective de construction d'un droit criminel inclusif.

MOTS CLÉS - Détermination de la peine, proches des contrevenants, conséquences de la peine, rationalité pénale moderne, liens sociaux.

1. Faculté des sciences sociales, Université d'Ottawa, 120, rue Université, pièce 5050, Ottawa (Ontario), Canada, K1N 6N5.

2. L'autrice tient à remercier Margarida Garcia, Dan Kaminski et Marie-Ève Sylvestre pour leur générosité dans la relecture d'une version préliminaire de ce texte, de même que les évaluateurs externes pour leurs suggestions et commentaires.

Criminologie, vol. 52, $\mathrm{n}^{\circ} 1$ (2019) 


\section{Introduction}

Ce qui n'est pas nommé n'existe pas. Ilhan Berk

La non-existence est produite chaque fois qu'une certaine entité est tellement disqualifiée qu'elle disparaît et devient invisible ou qu'elle est défigurée au point de devenir inintelligible.

Boaventura do Sousa Santos

Dans les tribunes politiques autant que dans les espaces médiatiques, les proches ${ }^{3}$ des personnes qui sont judiciarisées brillent par leur absence. Au-delà de l'«invisibilité sociale» (Lehalle, 2017, p. 21) qui caractérise cette population, la présente contribution va s'intéresser à leur «non-existence» pour les institutions juridiques, plus particulièrement à l'étape de la détermination de la peine. De quoi cette absence est-elle le nom? Quels effets produit-elle? Comment faire «exister» ces individus au regard du droit et des politiques publiques?

Dans Épistémologies du Sud (2011), le sociologue Boaventura de Sousa Santos a élaboré le canevas de ce qu'il appelle une sociologie des absences et des émergences. Cette sociologie «doublement transgressive» (p. 34) poursuit des objectifs décrits ainsi par Santos: la sociologie des absences vise à «rendre possibles les objets impossibles, [à] rendre présents les objets absents» (p. 34); la sociologie des émergences « consiste à remplacer ce que le temps linéaire présente comme le vide du futur (...) par des possibilités plurielles et concrètes, qui sont à la fois utopiques et réalistes» (p. 36).

Suivant l'invitation de Santos, cet article aura pour point de départ l'observation de l'absence et de l'émergence des proches des contrevenants lors de la détermination de la peine, et interrogera les rationalités sous-jacentes à l'invisibilisation de ces acteurs à cette étape du processus pénal ${ }^{4}$. Derrière la volonté de dévoiler l'absence, réside l'idée, suggérée par Santos, de «montrer que ce qui n'existe pas est en fait activement produit comme non existant, c'est-à-dire comme une alter-

3. Dans cette contribution, le terme «proches» sera utilisé au sens où l'entend Paugam (2013), soit en référence aux personnes entretenant un lien de filiation ou de participation élective (conjoints, amis et proches choisis) avec une personne judiciarisée.

4. Sans s'y limiter, l'étude du contexte canadien sera privilégiée dans cette contribution. 
native non crédible à ce qui est supposé exister» (2011, p. 34). En contrepoint, derrière la volonté de dévoiler l'émergence, réside l'idée, suggérée encore par Santos, d'«étend[re] le présent en ajoutant à la réalité existante les possibilités futures et les espoirs que ces possibilités suscitent» (2011, p. 37)

Pour répondre à ce programme, nous observerons d'abord la manière dont la théorie pénale moderne organise l'absence des proches des contrevenants, et réfléchirons aux rationalités œuvrant en coulisse pour construire et sédimenter cette absence. Nous verrons ensuite comment le droit positif (entendu ici comme étant constitué par les normes et la jurisprudence) organise à la fois l'absence et la présence des proches lors de la détermination de la peine. Nous proposerons enfin une réflexion sur la manière dont le dévoilement des absences et des émergences, qui viennent irriter les rationalités disqualifiantes pour les proches des contrevenants, pourrait contribuer à enrichir et modifier ces rationalités, dans la perspective de construction d'un droit criminel inclusif, qui serait plus ouvert à considérer les effets de la peine sur les liens sociaux.

\section{Le dépouillement social du contrevenant: une caractéristique du droit criminel moderne}

Si une perspective durkheimienne laisse supposer qu'une des fonctions symboliques de la peine réside dans le maintien de la cohésion sociale et de la stabilité des liens sociaux (Décary-Secours, 2016), cette fonction «fédératrice» prétendue, selon laquelle la justice pénale serait «le dernier rempart (...) utilisé pour protéger la société des atteintes graves [au] lien [social]» (Sanchez, 2012, p. 67), apparaît néanmoins en conflit avec les moyens que se donne l'intervention pénale pour atteindre ses objectifs. On constate ainsi que l'application du droit criminel peut contribuer paradoxalement à limiter, voire à entraver la cohésion sociale, et à fragiliser certains liens sociaux concrets ${ }^{6}$, notamment ceux que les contrevenants entretiennent avec leurs proches ${ }^{7}$.

5. Précisons toutefois que le propos de cet article se veut modeste; il s'agira d'ouvrir de nouvelles perspectives et d'amener une contribution à la réflexion émergente sur l'entourage des contrevenants.

6. Voir Mauer et Chesney-Lind (2002).

7. Voir Ricordeau (2008) et Touraut (2012). 
Avant d'examiner plus en profondeur la manière dont les institutions juridiques organisent l'absence des proches des contrevenants, nous nous interrogerons sur les sources de cette absence. À cet égard, deux éléments, intimement liés, apparaissent déterminants dans la construction d'un support cognitif isolant: d'abord, l'atomisme caractéristique des théories de la peine qui forment la rationalité dominante du système de droit criminel moderne (Pires, 2001a); ensuite, la centralité du contrevenant au moment de la détermination de la peine (Norrie, 2014), élément associé notamment à l'individualité de la peine.

L'atomisme des théories de la peine: un des attributs de la rationalité pénale moderne

Les contributions de la théorie de la rationalité pénale moderne (RPM) se sont avérées particulièrement riches pour observer le rapport entretenu par le système de droit criminel moderne à l'égard des proches des personnes judiciarisées, et ce, en particulier à l'étape de la détermination de la peine. Avant de décrire ce rapport, dressons d'abord un microportrait de cette théorie élaborée initialement par Alvaro Pires, et qui continue d'être ajustée au fur et à mesure de l'avancement des travaux menés notamment par Pires et par les membres de la Chaire de recherche du Canada en traditions juridiques et rationalité pénale.

La théorie de la RPM porte sur le système d'idées du droit criminel moderne; elle considère ce dernier comme un sous-système du droit, qui se différencie des autres sous-systèmes juridiques, et qu'on peut qualifier d'autonome (Pires, 2001a). Le coup d'envoi de la RPM fut donné par Beccaria avec l'ouvrage Des délits et des peines (1764/1991; voir Pires, 2008), et ce système d'idées s'est par la suite stabilisé en Occident autour de quatre théories de la peine: la dissuasion, la rétribution, la dénonciation et la réhabilitation en milieu fermé (Garcia, 2013). Les idées contenues dans ces théories «forment depuis le début de la modernité jusqu'à nos jours le «système de pensée» dominant du droit criminel moderne» (Garcia, 2013, p. 47), et cela malgré «quelques timides tentatives favorables aux alternatives» (Dubé, 2008, p. 24).

Quatre points de convergence caractérisent ces théories, quoiqu'à des degrés différents: 1) le droit de punir est perçu comme une obligation de punir ; 2) au sein de cette obligation, les peines afflictives et conduisant à l'exclusion sociale sont valorisées; 3) la peine de prison est présentée comme la «peine de référence»; 4) les sanctions alterna- 
tives à l'incarcération sont dévalorisées (Garcia, 2013). À travers ces caractéristiques fondamentales, la RPM constituerait «(...) un obstacle épistémologique (...) à l'innovation, c'est-à-dire à la création d'une nouvelle rationalité et d'une autre structure normative» (Pires, 2001a, p. 184$)^{8}$.

Le prisme fourni par la théorie de la RPM a permis de mettre en évidence quatre attributs de la peine valorisés par le système de pensée du droit criminel moderne (Pires, 2001a); nous insisterons ici sur l'un d'entre eux, soit le caractère atomiste de la peine. Pour Pires, la peine dans la RPM est «(...) atomiste, parce qu'[elle] (...) n'a pas à se préoccuper des liens sociaux concrets entre les personnes, sauf d'une façon tout à fait secondaire et accessoire» (2001a, p. 184). D'un point de vue conceptuel, le terme atomisme fait traditionnellement référence à une conception de la nature humaine qui part de la prémisse que l'individu est complètement autonome (Taylor, 1985), à tel point que «son identité n'est pas même en partie constituée par ses relations sociales» (Karmis, 1993, p. 85-86). Cette conception contraste avec la perspective aristotélicienne fondée sur l'idée que l'humain est par nature un animal social, qui ne peut se suffire à lui-même. Dominant la théorie politique moderne (Taylor, 1985), elle constitue une valeur traditionnelle de l'individualisme libéral (Mercure, 2005) et induit une survalorisation de la primauté des droits individuels; ainsi, les individus «n'ont pas l'obligation de soutenir la société et tous les membres de la société» (Pires, 2001b, p. 167).

Dès lors, l'atomisme caractéristique des théories classiques de la peine, mis en évidence par Pires, va induire une déconnexion entre la peine infligée et la vie sociale du contrevenant, ces théories faisant preuve d'une indifférence radicale à l'égard des liens sociaux entretenus par ce dernier:

les théories rétributive et dissuasive sont atomistes-belliqueuses, c'est-à-dire qu'elles ne prennent non seulement pas suffisamment en compte l'obligation morale et juridique envers la protection positive du lien social, mais qu'elles contribuent à la destruction de ce lien, créant un droit criminel de l'ennemi et une politique de «guerre froide». (Pires, 2001b, p. 167)

8. Ce constat ne doit toutefois pas être perçu comme fataliste: en effet, il «(...) n'implique pas l'absence d'idées ou de pratiques alternatives à la formation discursive dominante. (...). Des propositions alternatives existent (...) mais rien n'indique clairement que ces propositions ont été sélectionnées et moins encore stabilisées par le système de droit pénal moderne en Occident» (Cauchie et Kaminski, 2007, paragr. 9). 
Devenu un «ennemi de la société» (Pires, 2008), voire un «monstre» (Sylvestre, 2010), le contrevenant se voit alors exclu du monde social. Comme l'exprime bien Norrie (2014), «the ideologies of punishment constantly fail to see the social wood for the individual trees» (p. 363).

Cette rationalité atomiste, puissant support cognitif à la construction de l'absence des proches, contribue ainsi à anesthésier la pensée et permet au tribunal d'enfermer «un homme ou une femme en négligeant les liens affectifs qui le ou la constituent, au nom d'un raisonnement abstrait sur l'ordre social bafoué par le crime ou sur la sécurité de la société menacée par son auteur» (Kaminski, 2008, p. 1). Nettoyée de ses conséquences concrètes, la peine déterminée dans le contexte de la RPM pourra plus facilement faire l'impasse sur les dommages potentiels qu'elle engendrera sur le contrevenant, mais également sur son entourage.

\section{L'individualité de la peine}

Corollaire de cette rationalité atomiste, les théories de la peine modernes se sont ancrées originalement dans une fiction: l'individualité de la peine. Ce principe, à ne pas confondre avec celui de l'individualisation de la peine $^{9}$, constitue l'un des piliers du droit criminel moderne (Norrie, 2014), quoiqu'il ne soit pas codifié expressément dans le Code criminel canadien (ci-après: C. cr. $)^{10}$. Il se décline en deux temps: dans un premier temps, c'est la responsabilité individuelle du justiciable accusé qui devra être déterminée ${ }^{11}$, le refus de la responsabilité du clan pour un acte commis par un de ses membres apparaissant comme un "progrès» du droit criminel moderne. Dans un second temps, la sanction pénale est infligée à l'individu reconnu coupable, et la peine individuelle préside le droit criminel moderne. En effet, bien qu'on puisse observer, en marge de l'émergence du modèle réhabilitatif, un déplacement d'un individualisme abstrait vers une individualité concrète, les théories de la peine demeurent toutes centrées sur l'individu, quoique ce dernier y soit appréhendé de différentes manières (Norrie, 2014). Ainsi, même si dans certains pays et

9. Celui-ci fait généralement référence au fait que la peine est adaptée à la situation propre au contrevenant dans un cas donné.

10. Dans d'autres législations, on peut voir des traces de ce principe dans le droit positif, comme en France, où l'article 121-1 du Code pénal stipule que «Nul n'est responsable pénalement que de son propre fait».

11. Pour une réflexion sur l'émergence d'une forme de responsabilité collective dans la jurisprudence canadienne, voir Sylvestre (2013). 
cultures la punition collective existe toujours ${ }^{12}$, Christie (1986) remarque que celle-ci, "as of punishing some family member for the acts of others - have no great appeal in the value-systems of Western bumanity» (p. 97).

La détermination de la peine, arrimée à celle de la responsabilité criminelle, n'est donc officiellement plus collective. Toutefois, l'accent mis sur l'individu masque le fait que la peine n'atteint pas seulement celui ou celle à qui elle est publiquement infligée durant le rituel judiciaire. Le giron de la peine dite «individuelle» s'avère en réalité beaucoup plus large qu'il n'y paraît au premier abord, ce qualificatif contribuant à invisibiliser les dimensions collectives que la peine peut revêtir dans sa concrétude. Nous pourrions dire, de façon un peu provocatrice, que si la peine est déterminée individuellement, elle est, dans ses effets, purgée collectivement, engendrant des effets notables sur une panoplie d'autres acteurs (Comfort, 2007), quoiqu'elle ne les vise pas directement. Cette expérience collective de la peine, qui fait l'objet de ce numéro spécial, est pourtant bel et bien une réalité, qui a été mise en exergue notamment par Touraut (2013) sous le vocable d'«expérience carcérale élargie», expression qui «traduit l'emprise des prisons sur des personnes qui ne sont pas recluses et qui éprouvent pourtant quotidiennement la prison» (p. 94).

Ainsi, le principe de l'individualité de la peine comporte des effets occultants, et la centralité du coupable participe à la construction du mythe voulant que la peine prononcée ne touche qu'un individu; cette fiction contribue à produire l'absence de l'entourage du contrevenant, témoignant de l'abstraction du droit criminel moderne et de la déconnexion dont celui-ci fait preuve à l'égard des relations sociales concrètes entretenues par les individus. Or, comme le relève Bülow (2014), «no man is an island, and neither are offenders» (p. 776).

Si du point de vue du contrevenant, «(...) there is an un-traversable gap between the act of sentencing and the experience of suffering through punishment» (Berger, 2015, p. 21), cette remarque peut être étendue à l'expérience vécue par ses proches consécutivement au prononcé de la peine. En nous inspirant librement du raisonnement de Pires (2013), on peut dire que le système de droit criminel qui communique la peine doit et va s'appuyer sur des idées qui l'empêchent de voir ce qu'il est en train de faire; à cet égard, la centralité du contrevenant et l'atomisme

12. Voir par exemple Darcy (2003) sur la politique israélienne qui consiste à détruire les maisons des familles de Palestiniens ayant commis des actes qualifiés de terroristes. 
caractéristique des théories de la peine revêtent les traits de mécanismes de neutralisation quant à la visibilité des proches et des effets néfastes de l'intervention pénale sur ces acteurs. Or, une meilleure connaissance du contexte familial et social de la personne à qui la peine sera infligée permettrait, par hypothèse, de rendre plus visible le caractère radical de la peine de prison quant à son impact sur les liens sociaux. Comme le souligne Sylvestre (2010), en s'appuyant sur la notion de «totality of knowledge» évoquée par Christie (2000), la répression va sembler plus «raisonnable» lorsque l'on n'en sait pas trop sur la personne qu'on punit...

\section{L'organisation de l'absence et de la présence des proches par le droit}

Au Canada, la dislocation des familles qui peut suivre le prononcé d'une peine d'emprisonnement a été mise en évidence par plusieurs commissions de réforme: le Comité canadien de la réforme pénale et correctionnelle (commission Ouimet de 1969) a fait preuve d'un souci particulier eu égard aux «conséquences du procès, de la condamnation et de la peine sur la trajectoire de vie du délinquant [et] sur sa famille (...)» (Dubé, 2008, p. 3), et la Commission canadienne sur la détermination de la peine (commission Archambault de 1987) a souligné l'éclatement des familles et l'importance de leur préservation à cette étape du processus judiciaire. Pourtant, le cadre légal canadien relatif à la détermination de la peine demeure silencieux quant à l'existence des proches des contrevenants. Nous décrirons dans cette partie l'organisation différentielle de l'absence et de la présence des proches des contrevenants qui s'observe dans le droit positif canadien (normes et jurisprudence).

\section{Le silence de la loi: la cristallisation formelle de l'absence}

Alors que dans le contexte d'une peine infligée à une organisation, l'effet d'une peine sur la viabilité économique de l'entreprise et le maintien en poste de ses employés doit être considéré par le tribunal (art. 718.21d) C.cr.), aucune disposition comparable n'évoque les répercussions que la peine pourra engendrer sur les proches des contrevenants (pas plus que sur ce dernier) ${ }^{13}$. Notons que dans l'ensemble du

13. Cette remarque est valable à l'égard de l'ensemble des liens sociaux des contrevenants, mais le thème de cet article étant consacré aux proches, nous nous limiterons à 
cadre légal qui gouverne la détermination de la peine (art. 718 et suiv. C.cr.), c'est la seule disposition qui invite les tribunaux à considérer certains effets potentiels de la peine $\mathrm{e}^{14}$. La législation canadienne fait l'impasse sur l'existence des proches ${ }^{15}$, sauf pour aggraver la peine lorsqu'ils sont les victimes de l'infraction ${ }^{16}$, reconduisant par le fait même la rationalité isolante décrite précédemment. Les différents objectifs contenus dans les théories de la peine formant la RPM ont été «enchâssé[s] dans le Code criminel (...)» (Lachambre, 2013, p. 16) depuis la réforme de la détermination de la peine survenue en 1996; ces objectifs conduisent à sanctionner un individu isolé et dépouillé de son tissu social, invisibilisant par le fait même les tiers à la procédure. Ainsi, si les droits de la personne sont difficilement reconnus au coupable à l'étape de la détermination de la peine (Garcia, 2014), l'on peut se demander ce qu'il advient des droits des tiers qui pourraient être affectés par la peine.

La loi nous place ici devant un silence assourdissant, qui participe à une forme de normalisation des «coûts sociaux ${ }^{17}$ » d'envergure que le système pénal génère, à travers son fonctionnement, sur l'entourage des contrevenants. Cette normalisation découle d'une logique caractérisée par une absence marquée de réflexivité. Comme le remarque Desprez (2009), la réflexivité est la «réflexion se prenant elle-même pour objet», et ce terme «évoque la répercussion» (p. 481); or, le système pénal semble bien en mal de considérer les répercussions de ses interventions. Les principes de détermination de la peine insistent sur la nécessité de réparer le tort causé à la victime et à la collectivité par l'infraction (art. 718 a) et e) C.cr.), mais omettent de problématiser les torts causés par

ceux-ci. Pour un état des lieux de la prise en compte spécifique des enfants des contrevenants lors de la détermination de la peine, voir de Saussure (2017).

14. Les proches des contrevenants émergent néanmoins dans certains instruments internationaux. Voir par exemple les Règles des Nations Unies concernant le traitement des détenues et l'imposition de mesures non privatives de liberté aux délinquantes (Règles de Bangkok, 2011), et notamment les règles 58 et 64 , qui stipulent que les liens familiaux doivent être considérés lors du choix de la peine, et qui invitent à privilégier les peines non privatives de liberté pour les femmes enceintes et les femmes ayant des enfants à charge (cette règle s'appliquant également pour les hommes détenus aux termes du paragraphe 12 de cet instrument).

15. Cette remarque est également valable pour d'autres stades de la procédure pénale, notamment pour l'arrestation et la décision relative à la détention avant jugement.

16. Les articles 718.2 a) ii) et 718.2 a) ii).1 C.cr. créent des circonstances aggravantes si la victime est respectivement le conjoint ou l'enfant du contrevenant.

17. Expression empruntée à Landreville, Blankevoort et Pires (1981). 
la réaction opposée à cette dernière, qui apparaissent alors comme une tache aveugle du droit criminel.

Il est intéressant de noter que lorsque la personne condamnée est effectivement incarcérée, alors seulement ses proches apparaîtront comme sujets de normes relatives à l'exécution de la peine: la Loi sur le système correctionnel et la mise en liberté sous condition contient des dispositions relatives au maintien des liens (notamment familiaux) durant la détention ${ }^{18}$, et le Service correctionnel du Canada considère ce maintien «comme un facteur aidant la réinsertion sociale des détenus» (Lalonde, 2007). Ce report temporel de la prise en considération des proches, qui survient seulement à l'étape de l'exécution de la peine, répond logiquement au programme de la RPM qui survalorise la peine d'emprisonnement (Garcia, 2013), et à cette étape, les proches émergent suivant une rationalité servant la mission et les intérêts des services correctionnels. Une lourde responsabilité est attribuée aux familles, à qui l'on confère un rôle clé dans la réussite de la réinsertion du détenu (Touraut, 2013) ${ }^{19}$. Or, ce rôle ne leur est pas reconnu à l'étape de la détermination de la peine, alors que la réinsertion sociale constitue pourtant un des objectifs légaux de la peine aux termes de l'article 718 d) C.cr., et que la peine d'emprisonnement risque de «couper [le contrevenant] (...) de tous les liens sociaux qui pourraient encore stimuler plus positivement sa réhabilitation» (Dubé et Labonté, 2016, p. 709). Si, pendant la détention, l'importance de la place occupée par les proches «dans l'identification des stratégies de protection des liens familiaux» (Lehalle, 2017, p. 22) n'est pas suffisamment reconnue, cette remarque est tout aussi pertinente au moment d'infliger la peine.

\section{Des émergences observables dans la jurisprudence}

Alors que le cadre législatif est étanche aux effets de la peine sur les proches des contrevenants, les tribunaux ne font pas preuve de la même indifférence et se montrent plus pragmatiques, évoquant parfois ces effets dans leurs décisions. La problématisation de ces effets s'avère néanmoins diversifiée et non systématique. Nous aimerions relever ici

18. Ces dispositions visent la personne détenue, alors que ses proches ne bénéficient pas de droits corrélatifs codifiés. Voir par exemple l'article 71(1) de la loi, qui consacre le droit pour le détenu d'entretenir des rapports avec l'extérieur.

19. Ce rôle est également observé au moment de la remise en liberté dans la phase présentencielle, et dans le cas des peines non carcérales; voir Devresse (2012) pour les peines de travail et la surveillance électronique. 
les divergences conceptuelles, idéologiques et sémantiques observables dans la jurisprudence à cet égard. Nous décrirons ci-dessous trois «manières» de problématiser les effets de la peine sur les proches que nos recherches ont permis de mettre en exergue. Sans prétendre à l'exhaustivité ${ }^{20}$, les trois «manières» observées permettent néanmoins de dresser une description empirique intéressante de la problématique.

Les effets de la peine sur les proches: une conséquence du crime

Les tribunaux vont parfois considérer que les effets de la peine sur les proches découlent directement de l'acte délictuel, comme on peut l'observer dans R. v. Spencer (2004): "It is a grim reality that the young children of parents who choose to commit serious crimes necessitating imprisonment suffer for the crimes committed by their parents» (paragr. 46).

Cette problématisation s'inspire fortement de la logique rétributiviste kantienne, selon laquelle le contrevenant choisit sa peine en passant à l'acte, et partant, ses conséquences. Elle laisse apparaître une récupération du résultat matériel de l'acte, afin de légitimer le résultat matériel de la peine. Une telle imputation causale nous semble artificielle et mérite d'être déconstruite: c'est bien la peine, et non pas l'acte reproché, qui est à l'origine des effets de la peine, et une telle perspective, au-delà de la confusion qu'elle crée, conduit à déresponsabiliser le système pénal quant aux conséquences de son intervention. Le système est autonome par rapport à la transgression; la peine vient de lui, et non du condamné21. À cet égard, dans leur recherche relative aux «coûts sociaux du système pénal», Landreville et al. (1981) appellent justement à éviter l'amalgame entre ceux-ci et les «coûts du crime», insistant sur l'importance de distinguer la «logique du problème» de la «logique de l'intervention».

\section{Les effets de la peine sur les proches: une circonstance atténuante}

La deuxième manière de problématiser les effets de la peine sur les proches consiste à les classer dans la catégorie des «circonstances

20. L'analyse proposée a pour objectif de dresser un portrait des différentes problématisations observables, et non de rendre compte d'une analyse systématique de la jurisprudence rendue en matière de peine au Canada.

21. Cette affirmation s'oppose à une perspective hégélienne en matière de peine, selon laquelle la punition est voulue par le contrevenant et constitue son droit (voir Jodouin et Sylvestre, 2009). 
atténuantes», en application du principe de proportionnalité de la peine prévu à l'article 718.1 C.cr. ${ }^{22}$. Par exemple dans R. c. Bunn (2000), la Cour suprême du Canada a considéré comme atténuant le fait que le contrevenant doit pourvoir aux besoins de sa fille et de son épouse, qui était atteinte de la sclérose en plaques et invalide. Plus récemment, dans R. c. Landry (2016), le tribunal considéra comme des facteurs atténuants la relation de couple stable de l'accusé et le fait que ce dernier avait deux enfants ayant des problèmes de santé; il refusa d'appliquer la peine minimale obligatoire d'un an d'emprisonnement prévue pour l'infraction reprochée et la déclara contraire à l'article 12 de la Charte canadienne des droits et libertés, précisant qu'«une telle peine aurait des conséquences totalement disproportionnées sur l'accusé et sa famille» (paragr. 46). On peut néanmoins s'étonner du fait que c'est le «handicap» de santé des proches, déjà présent avant la peine, qui justifie l'atténuation, la peine proprement dite n'y étant pas envisagée comme handicap unique; dans ces exemples, c'est seulement lorsqu'elle est ajoutée à d'autres malheurs que la peine devient trop lourde pour les proches.

Les effets de la peine sur les proches: des conséquences dites «collatérales» ou «indirectes»

La troisième manière de problématiser les effets de la peine sur les proches consiste à les qualifier de conséquences indirectes ou collatérales de la peine. Elle a émergé consécutivement à l'arrêt $R$. c. Pham (2013), dans lequel la Cour suprême s'est penchée sur la prise en considération des conséquences de la peine en matière d'immigration. Bien que cet arrêt ne traite pas des conséquences sur les proches, il est venu apporter un éclairage intéressant quant à leur prise en compte, la Cour y problématisant la notion de «conséquences indirectes de la peine $»^{23}$ :

[11] (...) les conséquences indirectes découlant d'une peine s'entendent de tout effet qu'a celle-ci sur le délinquant concerné. Elles peuvent être prises

22. Ce principe actualise le rapport «crime-peine», impératif nécessaire présent dans toutes les théories de la peine de la RPM (voir Garcia, 2013; Pires, 2008).

23. Précisons que depuis le dépôt initial du manuscrit de ce texte, la Cour suprême a rendu l'arrêt $R$. c. Sutter (2018), dans lequel elle a précisé la définition applicable aux «conséquences indirectes de la peine». Nous n'entrerons pas dans les détails ici, mais le lecteur peut consulter notamment les paragraphes 46 à 49 de cet arrêt pour connaître la position la plus récente de la Cour à cet égard. Notons que dans cet arrêt, la Cour circonscrit ces conséquences à celles touchant le délinquant, tout comme dans Pham. 
en compte dans la détermination de la peine en tant que facteurs liés à la situation personnelle du délinquant. (...) [C]es conséquences ne constituent pas, à proprement parler, des facteurs atténuants ou aggravants, puisque, par définition, de tels facteurs se rattachent uniquement à la gravité de l'infraction et au degré de responsabilité du délinquant (...). Leur pertinence découle de l'application des principes d'individualisation et de parité. Les conséquences indirectes pourraient également être pertinentes à l'égard de l'objectif de la détermination de la peine qui consiste à favoriser la réinsertion sociale des délinquants (...). (nous soulignons)

Même si la Cour a circonscrit la définition des «conséquences indirectes de la peine» aux effets touchant le contrevenant, cet arrêt a été utilisé postérieurement pour soutenir la prise en compte des conséquences de la peine sur les proches de ce dernier. Par exemple, dans $R$. c. Stanberry (2015), le tribunal devait déterminer la peine à infliger à une femme ayant plaidé coupable à une infraction d'importation de cocaïne. Lors de l'audience sur la peine, Madame Stanberry a deux filles âgées de trois et sept ans, dont l'une présente une grave déficience auditive; elle représente la seule source de revenu et de soutien affectif pour ses filles. Le tribunal estime alors qu'il doit tenir compte des «conséquences collatérales» de la peine qu'il s'apprête à infliger et élargit la définition établie dans Pham:

[19] First, the evidence presented makes clear that Ms. Stanberry's children will be separated from each other for the duration of her term of incarceration. The effects of this fragmentation of the family in the development of young children, even long after the expiry of the term of imprisonment, are not subject to precise measurement: but they are certain to follow. Second, Ms. Stanberry will be separated from her younger daughter who suffers from serious medical difficulties that will have long-lasting effects.

[20] These two collateral consequences are not mitigating factors because they have no bearing on the gravity of the offence or the degree of the offender's responsibility. They are collateral in the sense that they are incidental to the terms of an appropriate sentence. In every other sense these collateral consequences will be direct and they will endure with great force not only for the duration of the term of imprisonment but beyond its expiry. They will entail what can only be described today as incalculable adverse effects both for the offender and her children. (...) (nous soulignons)

Sur le plan discursif, on voit apparaître dans ce jugement un élément inattendu, un «changement déviant» (Cauchie et Kaminski, 2007) par rapport à la rationalité atomiste présente usuellement dans les communications du système de droit criminel: les liens sociaux de la contre- 
venante sont ici visibles, et les conséquences qu'une peine de prison engendrerait sur ses proches sont reconnues. Toutefois, le tribunal met ensuite l'accent sur les objectifs de dénonciation et de dissuasion, et dévalorise la peine d'emprisonnement avec sursis (paragr. 25). La prise en compte des proches est ultimement ramenée à un calcul mathématique, soit une réduction du quantum de la peine, et une peine d'emprisonnement de 45 mois est infligée à Madame Stanberry. La prise en considération des proches n'a pas influé sur la nature de la peine octroyée, ce à quoi on aurait pu s'attendre dès lors que le tribunal évoque les risques concrets et «incalculables» associés à la fragmentation de cette famille.

Le même raisonnement s'observe dans plusieurs décisions subséquentes, où certaines théories de la peine semblent agir comme obstacle à une prise en compte conséquente des effets de la peine sur des proches $^{24}$, c'est-à-dire une prise en compte qui préserverait les liens que ces derniers entretiennent avec le contrevenant, et qui aurait un impact sur le choix du type de peine à privilégier. Sur le plan des idées, le support cognitif représenté par la mise en évidence des liens entretenus avec les proches s'avère insuffisant pour sortir de la logique de l'incarcération, leur préservation n'étant pas substantiellement valorisée par le système de droit criminel.

\section{Trois formes de problématisation, un seul résultat}

Indépendamment de la forme de problématisation endossée, on remarque que les effets de la peine sur les proches «opèrent en périphérie de la peine, dans les marges de l'activité judiciaire» (Parent et Desrosiers, 2016, p. 192). Précisons que ce constat ne doit nullement être interprété comme signifiant que les acteurs judiciaires ne se soucient pas des proches. En revanche, les idées qui dominent l'étape du choix de la peine, quoique non déterminantes eu égard aux pratiques, guident les acteurs en indiquant les possibilités valorisées en matière de sanction (Garcia, 2013), agissant alors comme barrière à une prise en compte substantielle de l'existence des proches. Ainsi, ces trois manières de problématiser les effets de la peine sur l'entourage des contrevenants semblent toutes aboutir à un résultat similaire: la reconnaissance de

24. Voir notamment R. c. Grondin (2015), R. c. Kaneza (2015), R. c. Brown (2015) et $R$. v. Zhou (2016), où les tribunaux mettent l'accent sur les objectifs de dénonciation et de dissuasion. 
l'importance des liens familiaux ne va pas nécessairement de pair avec leur protection, et les tribunaux semblent éprouver une certaine difficulté à utiliser substantiellement leur pouvoir discrétionnaire pour imaginer des solutions plus sensibles à la situation des proches. Enfin, bien qu'on ait récemment pu constater un essor des recherches relatives aux effets dommageables des peines de prison sur les familles, et en particulier sur les enfants ${ }^{25}$, notons que la jurisprudence ne semble pas évoluer d'une façon qui prendrait en compte ces «preuves empiriques» au moment de la détermination de la peine ${ }^{26}$.

\section{Imaginer le futur: les proches des contrevenants comme moteur d'un droit criminel inclusif}

Notre proposition dans ce texte vise à offrir des pistes de réflexion permettant notamment de construire, non seulement pour les proches des contrevenants mais également pour l'intervention pénale, un «Pas encore», au sens où l'entend Santos (s'appuyant lui-même sur les travaux d'Ernst Bloch): le «Pas encore» «exprime ce qui existe en tant que pure tendance, en tant que mouvement latent dans le processus même d'émergence. Le «Pas encore» est une façon d'inscrire le futur dans le présent et d'agrandir le présent»(Santos, 2011, p. 36-37). Dans ce sens, nous proposons d'inscrire la prise en compte des proches dans le cadre d'un droit criminel inclusif, qui valoriserait prioritairement le maintien du contrevenant dans la communauté, afin d'ancrer dans le système la possibilité de tenir substantiellement compte des proches, et d'en faire les artisans d'une réelle préservation des liens sociaux.

Pour que l'intervention pénale puisse s'en soucier, elle doit prendre place dans un modèle dialogique et réflexif, qui prend acte à tous ses échelons de la concrétude des interactions sociales, et qui reconnaît l'importance que chaque individu revêt pour sa communauté restreinte (famille, amis, proches), mais aussi pour l'ensemble de la collectivité, afin de le protéger contre une rupture sociale aux effets potentiellement dévastateurs. Or, une sanction qui fait preuve de respect à l'égard des

25. Voir Murray et Farrington (2008).

26. En revanche, de telles preuves ont été utilisées avec succès dans plusieurs affaires relatives aux conditions de détention: dans Inglis v. British Columbia (Ministry of Public Safety) (2013) quant aux programmes permettant la cohabitation mère-enfant en détention, ou encore dans British Columbia Civil Liberties Association v. Canada (Attorney General) (2018) quant aux mesures d'isolement cellulaire à durée indéterminée. 
liens sociaux n'est envisageable que si elle s'inscrit dans un contexte où les interactions sociales font sens pour le système. Comme le remarque Pires (2001b),

l'obligation envers le lien social ne se laisse pas obnubiler complètement par la transgression ni par la peine: elle exige la prise en compte de l'expression concrète, immédiate et positive des valeurs dans la réaction même qui veut les protéger. (p. 168)

Il importe également de rendre visibles et de problématiser les effets de la peine sur les proches dès sa détermination. Néanmoins, une mise en garde s'impose quant aux écueils possibles d'une telle mise en lumière: comme l'exprime justement Maggie Nelson (2017) dans Les Argonautes, si «[1]a visibilité concrétise, (...) elle discipline également» (p. 127). Rendre visible, dans le sens de montrer, a aussi pour effet de découper la réalité, de la définir. Ainsi, derrière l'éclairage que la visibilité jette, demeure une part d'ombre: parallèlement, son pouvoir peut aussi être celui de réduire. Elle peut être source de justification et de légitimation. Elle doit ainsi être révélée sans omettre ses effets potentiels. Autrement dit, les rationalités alternatives, tout comme l'argument critique émis à l'égard de la RPM, doivent également se montrer réflexives et soucieuses de leurs «répercussions». Par exemple, Wandall (2008) observe un profilage social répressif associé à la prise en compte de la situation familiale des contrevenants lors du choix de la peine: les personnes seules et isolées, sans relations sociales dignes de protection aux yeux du tribunal, peuvent s'en voir pénalisées, ce motif servant à justifier des décisions d'emprisonner ${ }^{27}$. L'auteur invite alors à opter pour une stratégie de détermination de la peine qui soit inclusive, y compris pour les personnes socialement marginalisées.

C'est ce fil qu'il s'agit selon nous de suivre, en réfléchissant à un mode d'intervention alternatif qui mette au cœur de son projet l'inclusion sociale, la préservation des liens sociaux, et également leur développement. À cet égard, la nouvelle autodescription du droit criminel ayant émergé dans le dernier rapport de la commission Ouimet, qui avait privilégié la théorie de la réhabilitation de la seconde modernité, nous semble encore d'actualité et particulièrement riche: «il s'agit de maintenir au premier plan l'inclusion, d'envisager différents types d'intervention (...) à l'intérieur de la communauté et de protéger concrètement les liens sociaux contre l'intervention destructrice du droit criminel» (Dubé,

27. Voir également Laberge, Landreville, Morin, Casavant et Charest (1998). 
2008, p. 55). Cette théorie marque clairement une rupture, étant «la seule à avoir échappé au dogmatisme de la rationalité pénale moderne et à avoir pu tracer les premiers contours d'un autre système de pensée» (p. 57). Comme le mentionne Pires (2012), les théories de la peine «restent indifférentes à l'inclusion sociale et ne proposent pas de «freins» ou de mécanismes d'autolimitation ou d'autocontention de la peine qui soient extérieurs aux caractéristiques du crime (...)» (p. 14-15). Or, les proches apparaissent justement comme un élément d'extranéité par rapport au crime; leur mise en lumière au moment du choix de la peine pourrait ainsi constituer un socle fécond pour penser autrement l'intervention pénale, en s'opposant à «la radicalité de l'indifférence» (Pires, 2012, p. 15) dont fait preuve le droit criminel à l'égard de la destruction des liens sociaux.

\section{Conclusion}

Dans cette contribution, nous avons observé les difficultés rencontrées par le droit criminel quant à la problématisation des effets de la peine à l'égard des proches des personnes contrevenantes. Ces derniers occupent une place ambivalente à l'étape de la détermination de la peine, entre absence et émergence. Nous avons également observé le blocage qui survient lorsque les tribunaux se montrent sensibles à l'existence des proches des contrevenants lors du choix de la peine: leur prise en considération s'avère concurrencée par le programme excluant de la rationalité pénale moderne, et les théories négatives de la peine viennent miner les éléments positifs associés à la présence des proches, empêchant de considérer leurs liens d'une manière qui les préserve substantiellement. La manière dont ces effets sont actuellement problématisés et «comptés» dans le cadre de la détermination de la peine demeure immobilisée dans le principe de proportionnalité des peines, qui s'arrime aux idéologies fondamentalement indifférentes au tissu social des individus, et valorisant l'exclusion sociale et l'affliction.

Ces difficultés appellent à problématiser ces effets autrement et précocement, en amont de l'exécution de la peine, afin qu'ils puissent être pris au sérieux par le droit et les politiques pénales. Néanmoins, une problématisation renouvelée demeurera insuffisante si elle s'inscrit dans la rationalité dominante du système de droit criminel moderne; en effet, cette contribution a mis en évidence le carcan dans lequel sont coincées les idées relatives à cette problématisation, soit une rationalité 
qui, en son cœur, construit déjà une prison cognitive autour du contrevenant, le dissociant de ses proches dès les balbutiements de la procédure. À cet égard, cette rationalité opère comme une barrière isolante, bridant les possibilités de prendre en compte les relations sociales des contrevenants dans la réflexion relative à la punition.

La problématisation des effets de la peine sur l'entourage des contrevenants doit ainsi s'ancrer théoriquement dans une rationalité alternative: les émergences survenant dans le cadre de la RPM parviennent difficilement à irriter cette rationalité, celle-ci ne présentant aucun support théorique et cognitif pour permettre une prise en compte des proches qui favorise l'inclusion sociale du contrevenant, et la préservation ou le développement de son tissu social. Cette rationalité doit nécessairement être remise en question pour qu'une problématisation s'avère transformatrice et générative d'idées nouvelles, au sens où l'entendent Garcia et Dubé (2017) : une problématisation qui puisse contribuer à penser «un nouvel idéal, (...) une utopie normative qui tout en étant autonome par rapport à celle du passé tient compte de ses écueils et de ses problèmes (...)» (p. 22). Il faut dessiner les contours d'un futur qui soit enrichi par les émergences observées dans cette contribution, et caractérisé par une relation d'apprentissage à l'égard du passé, et non de fidélité, comme le suggèrent Garcia et Dubé (2017). Il s'agit alors non seulement de «rendre visible» notre objet, mais également de le «rendre possible», comme Santos (2011) nous y invite.

\section{Références}

Beccaria, C. (1991). Des délits et des peines. Paris, France: Flammarion. (Ouvrage original publié en 1764.)

Berger, B. (2015). Sentencing and the salience of pain and hope. Osgoode Legal Studies Research Paper Series, 11(4), 1-23.

Bülow, W. (2014). The harms beyond imprisonment: Do we have special moral obligations towards the families and children of prisoners? Ethical Theory and Moral Practice, 17(4), 775-789.

Cauchie, J.-F. et Kaminski, D. (2007). Éléments pour une sociologie du changement pénal en Occident. Éclairage des concepts de rationalité pénale moderne et d'innovation pénale. Champ pénal, 4. Repéré à http://journals. openedition.org/champpenal/613

Christie, N. (1986). Images of men in modern penal law. Contemporary Crises, 10, 95-106.

Christie, N. (2000). Crime control as industry: Towards gulags, Western style (3e éd.). Londres, Royaume-Uni: Routledge. 
Comfort, M. (2007). Punishment beyond the legal offender. Annual Review of Law and Social Science, 3, 271-296.

Comité canadien de la réforme pénale et correctionnelle. (1969). Rapport du Comité canadien de la réforme pénale et correctionnelle. Justice pénale et correction: un lien à forger. Ottawa, Ontario: Imprimeur de la reine.

Commission canadienne sur la détermination de la peine. (1987). Réformer la sentence: une approche canadienne. Ottawa, Ontario: Ministre des Approvisionnements et Services Canada.

Darcy, S. (2003). Punitive house demolitions, the prohibition of collective punishment, and the Supreme Court of Israel. Penn State International Law Review, 21(3), 477-507.

Décary-Secours, B. (2016). L’«imaginaire pénal»: penser les significations sociales de la punition du côté des espaces sociaux marginalisés. Déviance et Société, 40(2), 227-244.

de Saussure, S. (2017). Quelle protection pour les enfants des contrevenants lors de la détermination de la peine? État des lieux au Canada. Dans J. Desrosiers, M. Garcia et M.-È. Sylvestre (dir.), Réformer le droit criminel au Canada / Criminal Law Reform in Canada (p. 433-458). Montréal, Québec: Éditions Yvon Blais.

Desprez, F. (2009). L'éventualité de mécanismes réflexifs dans le cadre du procès pénal français. Revue de droit de l'Université de Sherbrooke, 40(1-2), 479-509.

Devresse, M. S. (2012). Investissement actif de la sanction et extension de la responsabilité. Le cas des peines s'exerçant en milieu ouvert. Déviance et Société, 36(3), 311-323.

Dubé, R. (2008). Système de pensée et réforme du droit criminel: les idées innovatrices du rapport Ouimet (Thèse de doctorat, Université du Québec à Montréal). Repéré à https://archipel.uqam.ca/973/1/D1647.pdf

Dubé, R. et Labonté, S. (2016). La dénonciation, la rétribution et la dissuasion: repenser trois obstacles à l'évolution du droit criminel moderne. Les Cabiers de Droit, 57(4), 685-713.

Garcia, M. (2013). La théorie de la rationalité pénale moderne: un cadre d'observation, d'organisation et de description des idées propres au système de droit criminel. Dans R. Dubé, M. Garcia et M. R. Machado (dir.), La rationalité pénale moderne: réflexions théoriques et explorations empiriques (p. 37-72). Ottawa, Ontario: Presses de l'Université d'Ottawa.

Garcia, M. (2014). La géométrie normative variable des droits de la personne présents dans le système de droit criminel. Déviance et Société, 38(3), 361-385.

Garcia, M. et Dubé, R. (2017). La réforme du droit criminel: une idée dont le temps est venu. Dans J. Desrosiers, M. Garcia et M.-È. Sylvestre (dir.), Réformer le droit criminel au Canada / Criminal Law Reform in Canada (p. 3-35). Montréal, Québec: Éditions Yvon Blais.

Jodouin, A. et Sylvestre, M.-È. (2009). Changer les lois, les idées, les pratiques: réflexion sur l'échec de la réforme de la détermination de la peine. Les Cabiers de Droit, 50(3-4), 519-584.

Kaminski, D. (2008). «Editorial», Enfants de détenus, numéro spécial. Journal du Droit des Jeunes, (278), 1-2. 
Karmis, D. (1993). Cultures autochtones et libéralisme au Canada: les vertus médiatrices du communautarisme libéral de Charles Taylor. Revue canadienne de science politique, 26(1), 69-96.

Laberge, D., Landreville, P., Morin, D., Casavant, L. et Charest, R. (1998). Le rôle de la prison dans la production de l'itinérance. Collectif de recherche sur l'itinérance, la pauvreté et l'exclusion sociale. Repéré à https://depot.erudit. org/id/000793dd

Lachambre, S. (2013). L'évolution des objectifs de la peine en droit canadien. Dans E. Jimenez et M. Vacheret (dir.), Pénologie: réflexions autour de la peine et de son application (p. 13-31). Montréal, Québec: Les Presses de l'Université de Montréal.

Lalonde, N. (2007). Le maintien des liens familiaux lors de l'incarcération: une souffrance nécessaire? Actes du colloque Le pénal aujourd'hui: pérennité ou mutations (p. 229-242), Montréal, Québec.

Landreville, P., Blankevoort, V. et Pires, A. P. (1981). Les coûts sociaux du système pénal. Montréal, Québec: Université de Montréal.

Lehalle, S. (2017). Les proches: témoins, sujets, acteurs et experts de la sanction pénale malgré eux. Porte ouverte, 29(2), 20-22.

Mauer, M. et Chesney-Lind, M. (2002). Invisible punishment: The collateral consequences of mass imprisonment. New York, NY: The New Press.

Mercure, D. (2005). Libéralisme et lien social: une analyse critique. Cabiers internationaux de sociologie, 2(119), 333-346.

Murray, J. et Farrington, D. P. (2008). The effects of parental imprisonment on children. Crime and Justice, 37(1), 133-206.

Nelson, M. (2017). Les Argonautes. Montréal, Québec: Tryptique.

Norrie, A. (2014). Crime, reason and bistory. A critical introduction to criminal law ( $3^{\mathrm{e}}$ éd.). Cambridge, Royaume-Uni: Cambridge University Press.

Parent, H. et Desrosiers, J. (2016). Traité de droit criminel. Tome III: La peine ( $2^{\mathrm{e} e ́ d .) . ~ M o n t r e ́ a l, ~ Q u e ́ b e c: ~ E ́ d i t i o n s ~ Y v o n ~ B l a i s . ~}$

Paugam, S. (2013). Le lien social (3 éd.). Paris, France: Presses universitaires de France.

Pires, A. P. (2001a). La rationalité pénale moderne, la société du risque et la juridicisation de l'opinion publique. Sociologie et sociétés, 33(1), 179-204.

Pires, A. P. (2001b). «La ligne Maginot» en droit pénal: la protection contre le crime versus la protection contre le Prince. Revue de Droit Pénal et de Criminologie, 81(2), 145-170.

Pires, A. P. (2008). Aspects, traces et parcours de la rationalité pénale moderne. Dans C. Debuyst, F. Digneffe et A. P. Pires (dir.), Histoire des savoirs sur le crime et la peine, tome 2 ( $2^{\mathrm{e}}$ éd., p. 1-52). Bruxelles, Belgique: PUM-PUO-De Boeck Université, Collection Perspectives criminologiques.

Pires, A. P. (2012). Introduction. Les peines radicales: construction et «invisibilisation» d'un paradoxe. Dans I. Mereu (dir.), La mort comme peine (p. 7-47). Bruxelles, Belgique: Larcier.

Pires, A. P. (2013). L'énigme de l'échelle des peines en Occident: les droits de la personne, l'impunité et le paradoxe des "peines radicales». Document inédit. 
Ricordeau, G. (2008). Les détenus et leurs proches. Solidarités et sentiments à l'ombre des murs. Paris, France: Autrement.

Sanchez, F. C. (2012). Lien social émancipateur et modèles de justice? Pensée plurielle, 1(29), 61-74.

Santos, B. de S. (2011). Épistémologies du Sud. Études Rurales, 1(187), 21-49.

Sylvestre, M.-È. (2010). Rethinking criminal responsibility for poor offenders: Choice, monstrosity, and the logic of practice. Revue de droit de McGill, 55(4), 771-817.

Sylvestre, M.-È. (2013). The (re)discovery of the proportionality principle in sentencing in Ipeelee: Constitutionalization and the emergence of collective responsibility. Supreme Court Law Review, Osgoode's Annual Constitutional Cases Conference, 63, 461-481.

Taylor, C. (1985). Philosophy and the human sciences. Philosophical papers 2. Cambridge, Royaume-Uni: Cambridge University Press,

Touraut, C. (2012). La famille à l'épreuve de la prison. Paris, France: Presses universitaires de France.

Touraut, C. (2013). Aux frontières des prisons: les familles de détenus. Cultures $\sigma$ Conflits, 90, 77-94.

Wandall, R. (2008). Decisions to imprison. Court decision-making inside and outside the law. Hampshire, Royaume-Uni: Ashgate.

\section{Jurisprudence :}

Cour suprême:

R. c. Bunn, [2000] 1 RCS 183.

R. c. Pham, [2013] 1 RCS 739.

R. c. Suter, 2018 CSC 34.

Cour d'appel:

$R$. v. Spencer, 2004 CanLII 5550 (ON CA).

Tribunaux de première instance:

Inglis v. British Columbia (Ministry of Public Safety), 2013 BCSC 2309.

R. c. Stanberry, 2015 QCCQ 1097.

R. c. Grondin, 2015 QCCQ 8382.

R. c. Kaneza, 2015 ABQB 658.

R. c. Brown, 2015 ONSC 6430.

R. v. Landry, 2016 QCCQ 16626 (requête pour permission d'appeler sur la peine accueillie le 19 janvier 2017, puis désistement d'appel le 23 août 2017).

R. v. Zhou, 2016 ONSC 3233

British Columbia Civil Liberties Association v. Canada (Attorney General), 2018 BCSC 62.

\section{Textes légaux canadiens :}

Charte canadienne des droits et libertés (Loi constitutionnelle de 1982, Annexe B de la Loi de 1982 sur le Canada, R.-U.), 1982, ch. 11.

Code criminel (L.R.C. (1985), ch. C-46).

Loi sur le système correctionnel et la mise en liberté sous conditions (L.C. (1992), ch. 20). 


\section{Autres textes légaux :}

Code pénal (France). Repéré à https://www.legifrance.gouv.fr/affichCode.do? cidTexte=LEGITEXT000006070719

Règles des Nations Unies concernant le traitement des détenues et l'imposition de mesures non privatives de liberté aux délinquantes (Règles de Bangkok) (2011). Nations Unies, A/RES/65/229. Repéré à http://www.un.org/fr/ documents/view_doc.asp? symbol=A/RES/65/229

\section{The effects of punishment on the offenders' relatives: difficulties and discussion regarding their problematization at the sentencing stage}

ABSTRACT - This paper looks at the problematization of the effects of punishment on offender's relatives, specifically at the sentencing stage. To do this, we discuss the way modern criminal law organizes the absence of offenders' relatives and the reasoning that contributes to creating and solidifying this absence. We also look at how, in the background, some acknowledgment of the effects of absence takes place and affects the reasoning behind maintaining absence, reasoning that still dominates thought and practice in penal matters. Finally, we show that exposing the effect of absence and recognizing the possibility of overcoming it could contribute to informing and modifying this reasoning, making it possible to build a more inclusive system of criminal law.

KEYWORDS - Sentencing, offender's relatives, consequences of punishment, modern penal rationality, social ties.

\section{Las consecuencias de la pena sobre los familiares de los detenidos: dificultades y discusión en cuanto a su problematización en la etapa de la determinación de la pena}

RESUMEN • Este artículo se interesa por la problematización de los efectos de la pena sobre los familiares de las personas detenidas en el contexto de la determinación de la pena. Para observar este objeto, trataremos de la forma en la que el derecho penal moderno organiza la ausencia de los familiares de los detenidos y reflexionaremos sobre las racionalidades que trabajan tras bambalinas por la construcción y la sedimentación de esta ausencia. Veremos también cómo se organizan, en filigrana, los espacios de reconocimiento de los efectos de la pena sobre los familiares de los detenidos, y observaremos las emergencias que vienen a irritar a las racionalidades descalificantes hacia los familiares, racionalidades que dominan todavía el pensamiento y las prácticas en materia penal. Finalmente, propondremos una reflexión acerca de la manera en la que la confesión de las ausencias y de las emergencias, podría contribuir a enriquecer y a modificar estas racionalidades, dentro de la perspectiva de construcción de un derecho penal inclusivo.

PALABRAS CLAVE - Determinación de la pena, familiares de detenidos, consecuencias de la pena, racionalidad penal moderna, vínculos sociales. 